\title{
Electric Field Induced Alignment of Carbon Nanotubes: Methodology and Outcomes
}

\author{
Ali Mohammad Amani, Seyyed Alireza Hashemi, \\ Seyyed Mojtaba Mousavi, \\ Seyyed Milad Abrishamifar and Arash Vojood
}

Additional information is available at the end of the chapter

http://dx.doi.org/10.5772/intechopen.70481

\begin{abstract}
In the current chapter, achievement of aligned carbon nanotube (CNT) network within the matrix via various kinds of electric fields (AC and DC) was evaluated. In this case, alignment mechanism of CNTs within the matrix and two useful techniques for justification of CNT alignment throughout the matrix were examined and presented, respectively. Afterward, effective factors in matter of CNT alignment and applicable procedures for fabrication of nanocomposites containing aligned CNTs were studied and presented, respectively. At the end, significant effects of CNT alignment on overall properties of nanocomposites that include electrical and mechanical properties were evaluated. Achieved results revealed that alignment of CNTs within the matrix can lead to significant improvement in the electrical and mechanical properties of nanocomposites at the same filler loading compared with randomly distribution of CNTs within the matrix, while production steps and conditions can also highly affect the outcome data.
\end{abstract}

Keywords: carbon nanotube, nanocomposite, electric field, alignment, electrophoresis

\section{Introduction}

Carbon nanotube (CNT) is a unique material with fantastic electrical, thermal, and mechanical properties, and since its discovery by Iijima in 1991 [1], global attention attracted toward it, and many researchers have evaluated the extraordinary properties of CNTs toward development of nanocomposites and sheets holding highly oriented CNTs with enhanced electrical and mechanical properties [2-7]. 
Alignment of CNTs within the matrix can boost their properties in various fields include electrical and mechanical properties [2-7]. What is more, alignment of CNTs can be achieved via various methods such as electrical field $[2-4,7,8]$, magnetic field [9], shear flow [10, 11], mechanical force [12, 13], and electrospinning [14, 15], which electrical fieldinduced alignment is the simplest and most manageable method for alignment of CNTs within the matrix.

Besides, exert of electrical field to a matrix containing CNTs can lead to expansion of a highly oriented network from the negative electrode toward the positive electrode, which acting as a pathway for transferring current from the negative electrode toward the positive electrode. In this case, interruption of applied current can lead to collapse of developed network which known as relaxation mechanism [16].

In this chapter, first the alignment mechanism of CNTs within the matrix was evaluated, and thence, useful techniques for justification of CNT alignment in the content of matrix were presented. Afterward, effective techniques for fabrication of nanocomposites containing highly and randomly oriented CNTs were discussed, and effect of alignment on various properties of nanocomposites was examined.

\section{Alignment mechanism of CNTs within the matrix}

Alignment mechanism of CNTs consisted from four stages [16]. In the first stage, due to the application of electric field, a dipole moment induced at the edges of CNTs resulted in their rotation to a certain angle and thence aligned in the direction of electric field. In the second stage, polarized CNTs attract each other, and head-to-head contact occurs, forming an aggregating in a chain-like structure. In the third stage, CNTs migrate toward and attach to the negative electrode, respectively. When CNTs are close enough to the electrode in order to transfer the charge, they discharge and aggregate onto the electrodes. Attached CNTs to the electrode become sources of high field strength and primary locations for absorption of other CNTs. In the fourth stage, following the connection of the first CNT bundle to the negative electrode, other CNT bundles attached the first bundle, and thence aligned CNT network spans negative-positive electrode spacing. In fact, CNTs form a pathway for transferring the current from the negative electrode toward the positive electrode and if the electric field interrupted during the alignment process, highly oriented network breaks down and CNTs return to their primary locations, this phenomenon is known as relaxation mechanism $[16,17]$. Likewise, the translational motion of CNTs toward the negative electrode, justifying the presence of a negative surface charge, is governed by the electrophoretic mobility of charged CNTs. In case of relaxation mechanism, the Brownain diffusion is relevant to the motion and tends to fade of the aligned network, till it returns to the primary randomly distribution situation [18]. In Figure 1, alignment process of CNTs within matrix can be seen. 


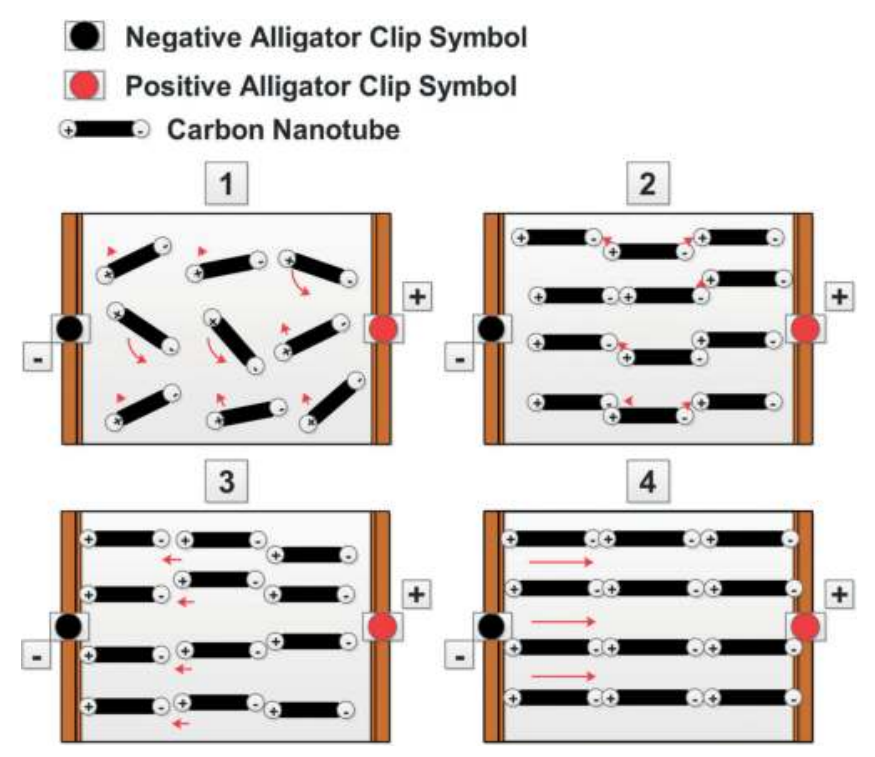

Figure 1. Alignment behavior of CNTs under application of electric field, (1) rotation, (2) head-to-head contact, (3) migration toward the negative electrode and (4) formation of aligned network between electrodes.

\section{Justification of CNT alignment}

There are several effective techniques for evaluation of CNT alignment within the matrix. In this case, the most common technique is via micro Raman spectroscopy. Raman spectrum of $\mathrm{CNT} /$ polymer nanocomposites features two characteristic peaks, namely D-band and G-band. The band at $1350 \mathrm{~cm}^{-1}$ is known as D-band and corresponds to overall amount of defects in carbon atom bonds in curved graphene sheets. Therefore, the structural defects including heteroatoms, vacancies, impurities, as well as pentagon-heptagon pairs in helical structures of CNTs can activate the D-band feature. The other strong band observed at $1590 \mathrm{~cm}^{-1}$, called G-band, corresponds to the graphitization/crystallization degree of CNTs. G-band is believed to derive from the stretching of $\mathrm{sp}^{2}$-hybridized carbon atom bonds along the surface of graphitic CNTs [19-21]. Alignment of CNTs within the polymer matrix has significant effect on both D-band and G-band peaks [22-24]; this introduces Raman spectroscopy as a complementary technique to verify the alignment of SWCNTs within the polymer matrix.

For this purpose, $D(G)$ band intensities obtained parallel and perpendicular to CNT alignment direction are denoted as $D_{\|}\left(G_{\|}\right)$and $D_{\perp}\left(G_{\perp}\right)$, respectively. Enhancement of $D_{\|} / D_{\perp}$ and $G_{\|} / G_{\perp}$ values corresponds to improvement of CNT alignment within the matrix, due to higher surface area of aligned CNT projected to applied laser light in parallel direction than perpendicular direction. Abbasi et al. [19] and Arjmand et al. [20, 25, 26] used this technique in order to examine the 
alignment degree for compression-molded and injection-molded multi-walled carbon nanotube (MWCNT)/polymer nanocomposites. Their obtained results revealed that alignment of MWCNT via injection molding method had higher $D_{\|} / D_{\perp}$ and $G_{\|} / G_{\perp}$ values compared to the compressionmolded samples, which contain randomly distributed CNTs. They also found out that D-band is more sensitive to MWCNT alignment than G-band; thus, higher values of $D_{\|} / D_{\perp}$ than $G_{\|} / G_{\perp}$ are expected for the aligned nanocomposites compared to unaligned nanocomposites.

The higher the ratio of $G_{\|} / G_{\perp}$ the better the quality of aligned CNT network [27]. In a study by Chapkin et al. [28], they have developed a technique, which has utilized polarized Raman spectroscopy for evaluation of CNT alignment within the polymer matrix under applied electric field. In this case, the effect of electric field strength between range 200 and $1100 \mathrm{~V} / \mathrm{cm}$ on the degree of alignment and required time for achieving highly oriented CNT network was examined. The Raman scattering intensity is proportional to falling CNTs within the excitation volume of the laser. Increase in the concentration of CNTs within the matrix not only can increase the likelihood of multiple scattering events but also can increase the opacity of the mixture, which can lead to decrease in the penetration depth of the laser. Comparison between intensities of Raman spectra provided by orthogonally polarized incident light in nanocomposites containing wellaligned CNTs is an evaluation method for alignment characterization. Electrostatically aligned network of CNTs can lead to increase in the G-band intensity for parallel polarization direction and decrease for perpendicular direction in comparison to unaligned network. The $G_{\|} / G_{\perp}$ ratio indicating the degree of CNT alignment, which increases in this ratio, is the sign of better alignment within the matrix. In an unaligned specimen, this ratio is approximately 1 . By applying the electric field between electrodes and alignment of CNTs between them, the intensities related to Raman peaks in the CNT spectrum begin to rise. In this case, the intensity of G-band was increased for the parallel polarization and decreased for the perpendicular polarization than unaligned specimens. Besides, by interruption of electric field, a considerable drop in the normalized G-band intensity was observed which is known as relaxation mechanism [16]. Decrease in the G-band intensity due to interruption of electric field indicating the loss of CNT alignment and collapse of aligned network. Furthermore, viscosity of matrix can highly affect the alignment behavior of CNTs. In this regard, a rapid loss in CNT alignment on the order of tens of milliseconds observed for low viscosity systems by interruption of the electric field [29, 30]. However, it has been observed that the alignment degree of CNTs in a matrix with high viscosity is higher than in a matrix with lower viscosity [31]. Increase in the electric field strength can either improve the $G_{\|} / G_{\perp}$ ratio or degree of alignment within the matrix. On the other hand, the polarization of a CNT highly depends on its conductivity [32,33] and aspect ratio [32]. Conductivity of a CNT is based on its charity and behavior, which could be metallic or semiconducting. It has been proved that metallic CNTs presenting a greater degree of alignment via applied electric field than semiconducting CNTs [28]. Khan et al. [3] and Chen et al [4] have used micro Raman spectroscopy for determining the degree of CNT alignment via applied DC and AC electric fields, respectively.

In addition, Monti et al. [16] and Martin et al. [17] have measured the electrical current during the exertion of electric field as an indicator for formation of a highly oriented network in the area between two opposite electrodes. In fact, when highly oriented CNT network completed, the negative surface charge on the surface of the suspension has become constant, which shows highly oriented network has completed. Moreover, this network is a conductive 

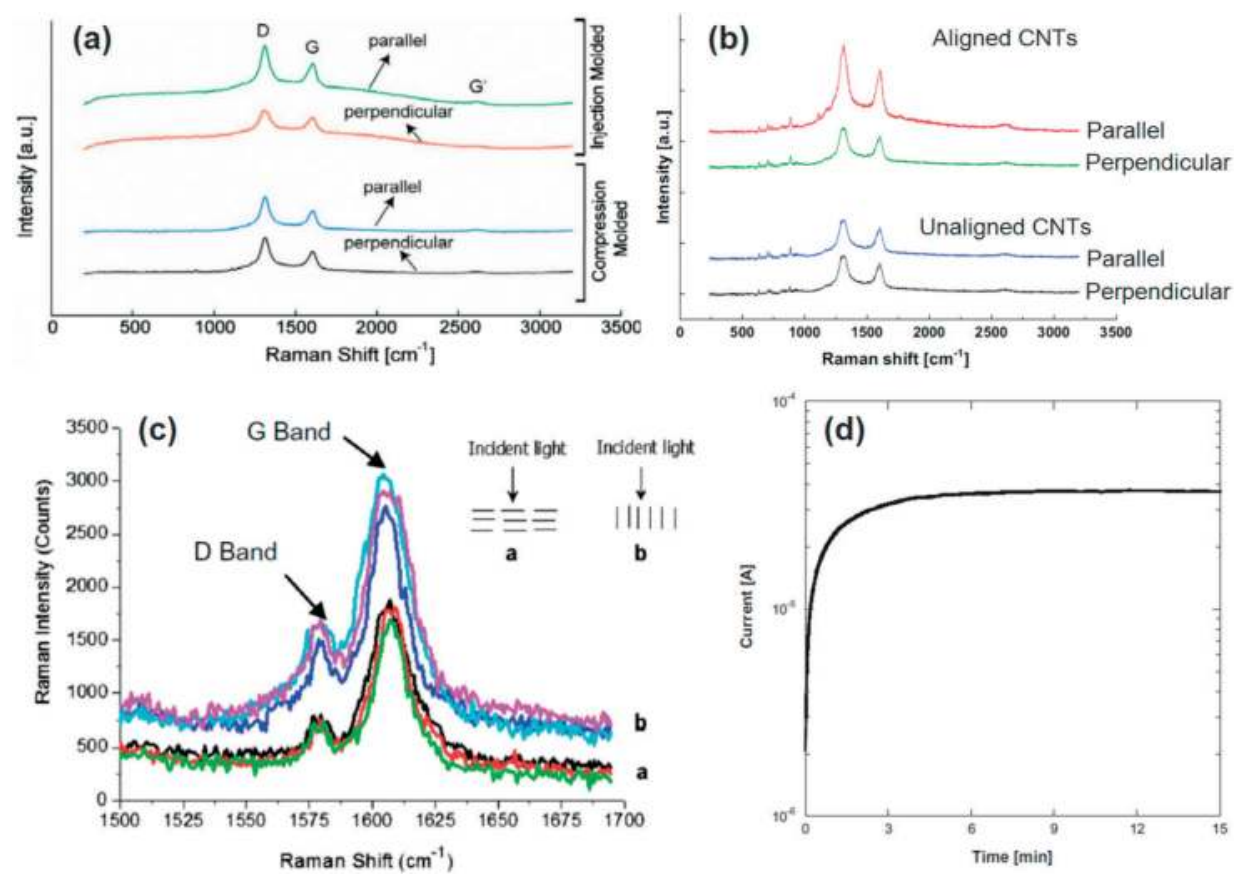

Figure 2. (a-c) Justification of CNT alignment via micro Raman spectroscopy [3, 19, 20], (d) Justification of CNT alignment via constant current on suspension surface [16].

path for transferring the current toward the positive electrode under electrophoresis, which verifying the presence of negative surface charge on CNT surfaces [17]. In Figure 2, a view of various justification methods for CNT alignment can be seen.

\section{Effective factors on CNT alignment}

Many effective factors involved in case of CNT alignment, which evaluation of their effects on aligned network is very essential. Homogenous dispersion of pristine CNT in the matrix is difficult to achieve, which is due to the poor interfacial interaction between CNTs and matrix $[34,35]$. What is more, functionalization of CNTs can improve their dispersion within the matrix and enhance the align network. The alignment of CNTs within the matrix has significant effect on overall properties of developed nanocomposites, which are mainly dependent on the degree of CNT dispersion, type of matrix, and interfacial bonds between the matrix and fillers [3]. On the other hand, functionalization of CNTs can improve their dispersion and thus alignment of CNTs within the matrix due to the better interaction with matrix. In fact, functionalization of CNT can change its nature from hydrophobic to hydrophilic [36]. But functionalization of CNTs can increase their resistivity and examination of nanocomposites containing aligned CNTs revealed that highly oriented network consisted form pristine CNTs 


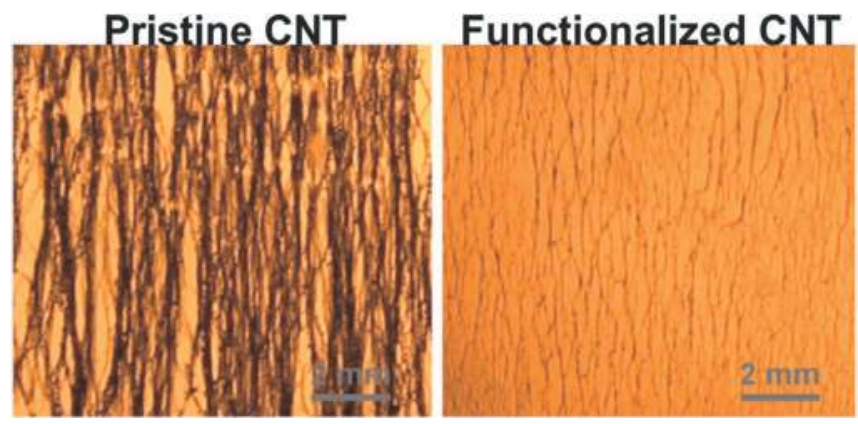

Figure 3. Comparison between performance of pristine and functionalized CNTs for formation of aligned network under application of AC electric field [4].

presenting higher electrical conductivity than functionalized CNTs [8]. A view of aligned CNTs networks containing pristine and functionalized CNTs can be seen in Figure 3. As can be seen in this figure, pristine CNTs have formed agglomerated bundles, while functionalized CNTs formed approximately homogenous aligned network.

Furthermore, alignment of CNTs within the matrix can be achieved via both AC and DC electric fields, while behavior of CNTs highly depends on the type of employed electric field and surface charge on the surface of suspension $[17,37]$. In regard of DC electric field, migration of CNTs toward the negative electrode is governed by their electrophoretic mobility, which highly depends on the surface charge, while the electrophoretic mobility for AC electric field is zero. This specification of AC electric field can lead to creation of homogenous aligned network within the matrix due to their dielectrophoretic behavior. Likewise, usage of AC electric field found to be more effective compared with DC electric field [4, 17]. Moreover, increase in the electric field voltage can enhance the degree of CNT alignment, while for both AC and DC electric fields, increase in the electric field voltage can increase the aggregation rate, which highlights the requirement for optimization of voltage level [3, 8]. It was revealed that increase in the frequency of AC electric field can enhance the alignment degree and increase the transvers connections between aligned CNT bundles in the direction of electric field [8]. On the other hand, AC electric field presented both better dispersion and alignment within the matrix compared with DC electric field [4]. In Figure 4, effect of CNT weight percentage and DC electric field exerting time and strength on the quality of final aligned network can be seen. As can be seen in this figure, increase in CNT weight percentage and voltage can lead to increase in agglomeration rate and creation of robe-like CNT bundles which can be seen in Figure 4(g). Moreover, a view of aligned CNT network in fully cured samples can be seen in Figure 5.

In addition, applied voltage and viscosity of matrix can highly affect the alignment process. Increase in the voltage and decrease in the viscosity of matrix can decrease the alignment time. However, unnecessary increase in the electric field voltage can improve the aggregation rate of CNTs and lead to creation of rope-like thick CNT bundles. On the other hand, due to high aspect ratio of CNTs, longitudinal polarization overcomes the transverse polarization. It is worth noting that after completion of the longitudinal polarization, CNTs start to attach each other in the transverse direction, forming transverse crosslinking [3, 16]. In Figure 6(a-c), effect of viscosity 


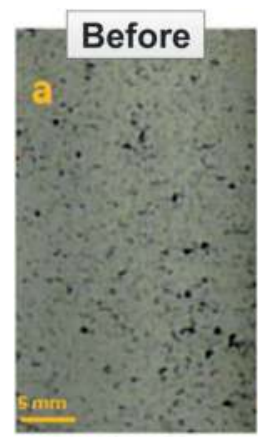

$0.05 \%$ CNT

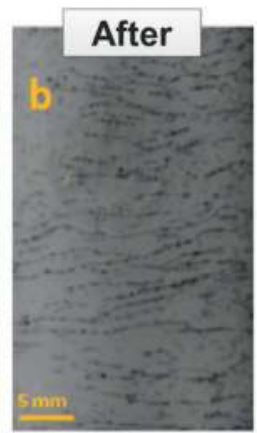

$0.05 \%$ CNT

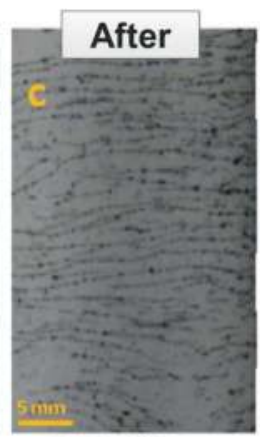

$0.1 \%$ CNT

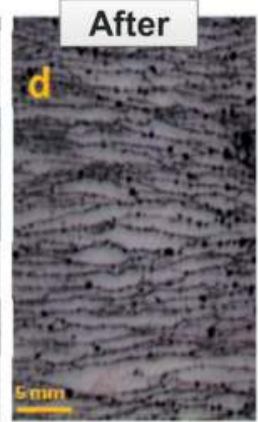

$0.15 \%$ CNT

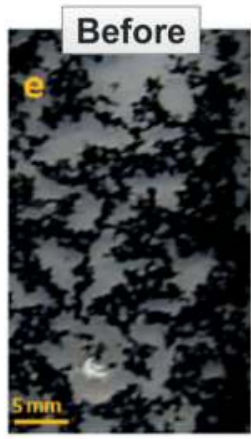

$0.1 \%$ CNT

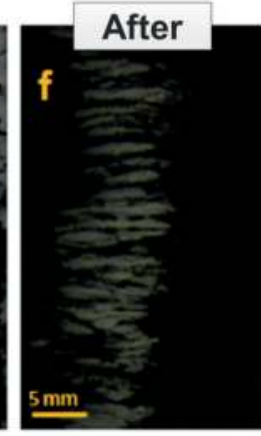

$0.1 \%$ CNT

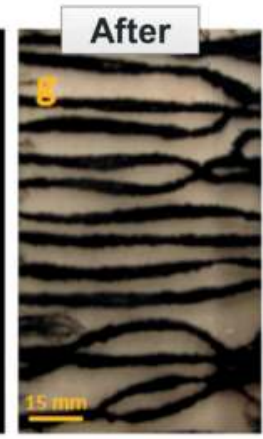

$0.05 \%$ CNT

Figure 4. Optical images from aligned CNT networks with respect to their concentration, size and duration which DC electric field was applied between electrodes, (a-f) $100 \mathrm{~V} / \mathrm{cm}$ for $30 \mathrm{~min}$ and (g) $200 \mathrm{~V} / \mathrm{cm}$ for $30 \mathrm{~min}$ [3].
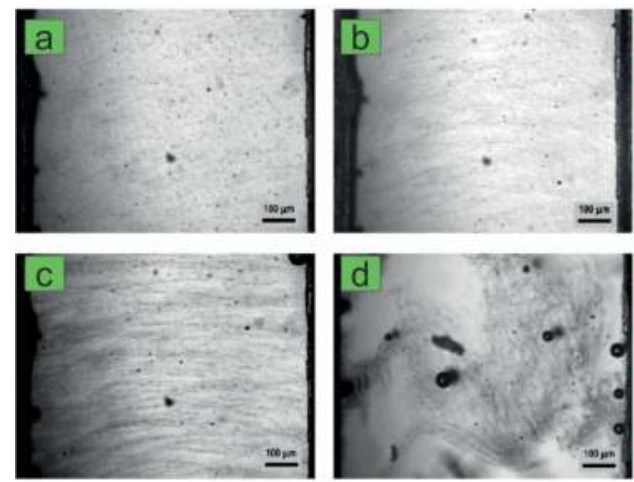
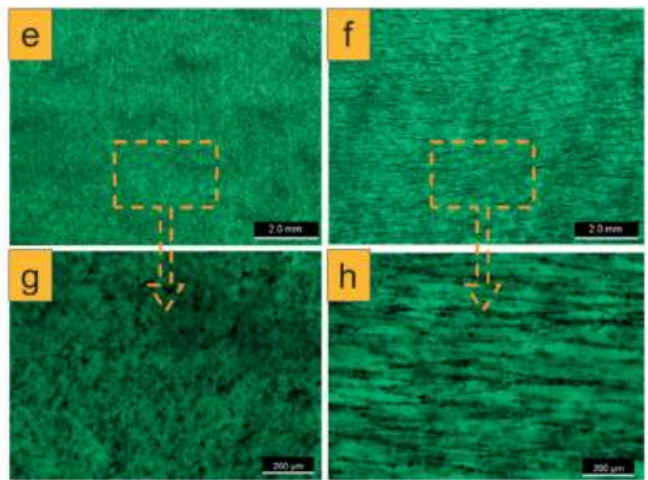

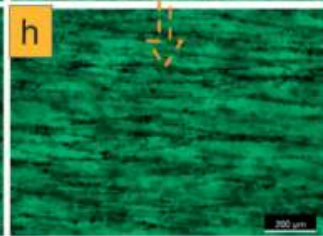

Figure 5. Optical micrographs of suspension containing (a) randomly distributed SWCNTs before the field application, (b) alignment of SWCNTs after $180 \mathrm{~s}$ of field application, (c) alignment of SWCNTs after 15 min of field application, (d) a view of relaxation mechanism 10 min after switching the electric field off [16], (e and g) random distribution of MWCNTs within the matrix and ( $\mathrm{f}$ and $\mathrm{h}$ ) alignment of MWCNTs within the matrix via DC electric field [3]. 


\section{(a) Rotation}

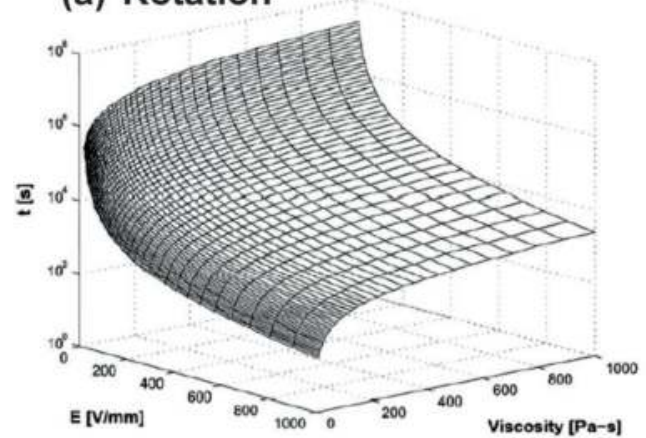

(c) Migration

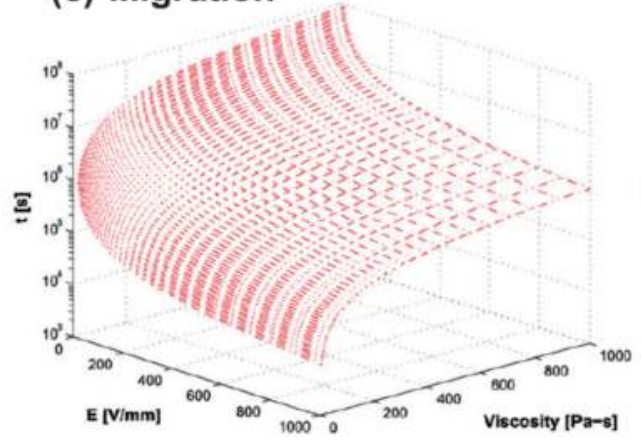

(b) Chain-like structure formation
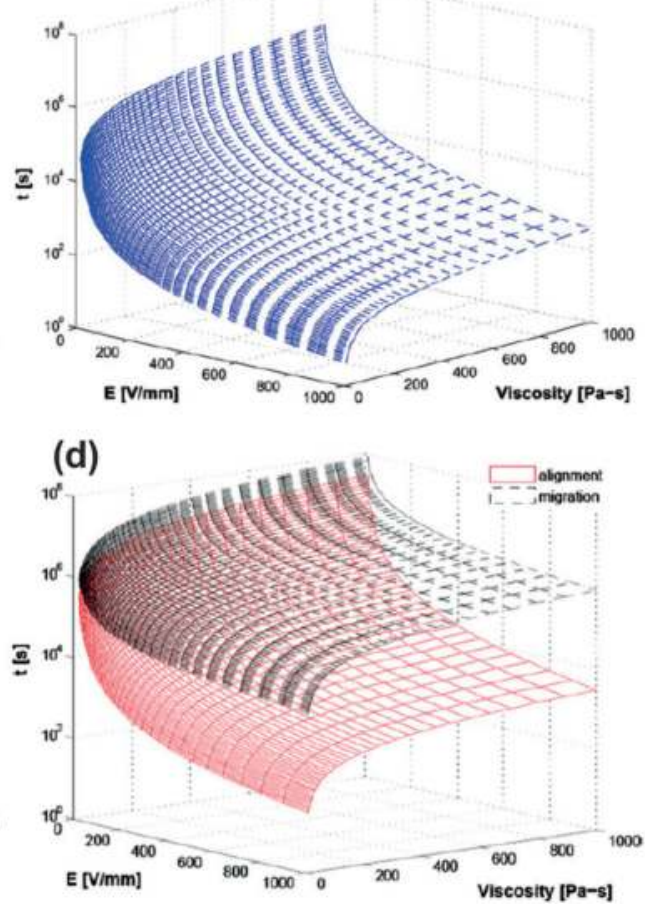

Figure 6. Effect of electric field strength and viscosity of matrix on the required time for (a) rotation, (b) head to head contact and (c) migration, (d) shows comparison between required time for alignment of CNTs within the matrix and migration of CNTs toward negative electrode [16].

and electric field strength on required times for rotation, formation of chain-like structure, and migration can be seen, respectively. Besides, in Figure 6(d), comparison between total alignment time and required time for CNT migration toward negative electrode can be seen.

\section{Methodology}

In order to achieve a nanocomposite containing highly oriented CNT network with homogenous distribution, development of a practical procedure which has considered all of effective factors in matter of CNT alignment and nanocomposites preparation is essential. In this case, Ma et al. [4] synthesized MWCNTs using chemical vapor deposition (CVD) technique and thence functionalized fabricated MWCNTs using acidic method. In this regard, a mixture of $\mathrm{H}_{2} \mathrm{SO}_{4}$ and $\mathrm{HNO}_{3}$ acid (1:1 volume ratio) was prepared, and thereon, MWCNTs were added to the previous solution and heated up to $150^{\circ} \mathrm{C}$ for $30 \mathrm{~min}$. Then, MWCNTs were rinsed with deionized water till they become chemically neutral. FTIR examination revealed that these MWCNTs have hydroxyl $(-\mathrm{OH})$ and carboxyl $(-\mathrm{COOH})$ functional groups, which 
can improve their dispersion within the matrix. Thence, MWCNT/poly methyl meth acrylate (PMMA) composites fabricated using in situ polymerization technique. In this regard, $0.1 \mathrm{wt} \%$ 2,2-azobisisobutylonitrile (AIBN) was dissolved in liquid methyl methacrylate (MMA) monomer. Then, desirable weight percentage of MWCNTs added to the MMA/AIBN solution and ultrasonicated for $15 \mathrm{~min}$. In the next step, in order to align MWCNTs in the matrix, AC voltage of $300 \mathrm{Vp}$-p (peak to peak) at $500 \mathrm{~Hz}$ was maintained across the copper electrodes. The resulting nanocomposite was allowed to cure under the AC electric field for $2 \mathrm{~h}$ at $70^{\circ} \mathrm{C}$.

Zhu et al [7] developed nanocomposites containing aligned MWCNTs within the epoxy resin. In this regard, MWCNTs were synthesized with mean diameter and average length of $20 \mathrm{~nm}$ and $10 \mu \mathrm{m}$, respectively. Afterward, they have developed two different kinds of MWCNT. For the first MWCNT type, MWCNTs were purified with hydrofluoric acid for $24 \mathrm{~h}$ in order to remove impurities and residual catalyst from synthesized MWCNTs. Thence, purified MWCNTs were placed in an oven at $100^{\circ} \mathrm{C}$ for $24 \mathrm{~h}$, thereby resulting in production of pristine MWCNT. For development of second type of MWCNTs, pristine MWCNTs were added into a solution of $\mathrm{H}_{2} \mathrm{SO}_{4}$ and $\mathrm{HNO}_{3}$ (volume ratio 1:1) and resulting suspension heated up to $150^{\circ} \mathrm{C}$ for $30 \mathrm{~min}$. Thereon, MWCNTs were filtered and rinsed continuously with deionized water in order to make it chemically neutral. This procedure can lead to production of functionalized MWCNTs with carboxyl $(-\mathrm{COOH})$ and hydroxyl $(-\mathrm{OH})$ functional groups. the oxidized MWCNTs were thence refluxed in thionyl chloride at $80^{\circ} \mathrm{C}$ for $1 \mathrm{~h}$, and residual suspension was distilled and added into a mixture of methylene dichloride, 1,2-ethylenediamine, and triethylamine. Resulting suspension was stirred for $1 \mathrm{~h}$ at room temperature. Eventually, MWCNTs were filtrated out, rinsed with deionized water till become chemically neutral, and then dried at $100^{\circ} \mathrm{C}$ for $24 \mathrm{~h}$ to gain amine functionalized MWCNT. In case of matrix, they have used an epoxy resin which could be cured via emission of UV beams and while irradiated by $265-n m$ UV light, it was fast polymerized within several minutes. In the next stage, specific amount of prepared MWCNTs was poured in the epoxy resin and ultrasonicated for $20 \mathrm{~min}$. Then, $2 \mathrm{~mL}$ of the resulting suspension poured into the related mold, and thence, an AC voltage of $2000 \mathrm{Vp}$-p (peak to peak) at $200 \mathrm{~Hz}$ was applied to the suspension for approximately $10 \mathrm{~min}$. Then, UV light was emitted to the suspension for $10 \mathrm{~min}$, which resulted in fast polymerize (cure) of the suspension. A view of their fabrication procedure can be seen in Figure 7(a).

Khan et al. [3] used CVD fabricated MWCNTs for development of nanocomposites containing highly oriented CNTs. For this regard, first MWCNTs were subjected to ultra-violet/ozone $\left(\mathrm{UV}-\mathrm{O}_{3}\right)$ treatment for $30 \mathrm{~min}$ used equipment consisted from a low-pressure mercury vapor grid lamp, which could irradiate UV radiation of $28 \mathrm{~W} / \mathrm{cm}^{2}$ to samples placed $20 \mathrm{~mm}$ far from it. This step can change the nature of CNTs from hydrophobic to hydrophilic due to the creation of oxygen-based functional groups on CNT surface, thereby enhancing the interaction between matrix and CNTs. Functionalized MWCNTs sonicated in acetone and Tritone $\mathrm{x}-100$. Thence, epoxy resin was added to the previous suspension and sonicated for further $1 \mathrm{~h}$. Thereon, CNT/epoxy mixture was placed in the vacuum oven for $2 \mathrm{~h}$ in order to evaporate acetone from the suspension. For further degassing, suspension was passed through a threeroller mill for six times. Resulting mixture was then degassed for further $20 \mathrm{~min}$ to eliminate trapped air bubbles, and thereupon, curing agent triethylenetetramine was added to the epoxy 
resin with ratio 12:100. MWCNTs aligned due to application of DC electric field in a place between two aluminum electrodes with $50 \mathrm{~mm}$ distance. In this case, 100 or $200 \mathrm{~V} / \mathrm{cm}$ electric field was applied to the mixture depend on the concentration of MWCNTs. An elevated temperature of $100^{\circ} \mathrm{C}$ was applied to the mixture after completion of alignment, which resulted in the rapid cure of the mixture. Primary cured composite was then postcured at $120^{\circ} \mathrm{C}$ for $2 \mathrm{~h}$. A view of their production technique can be seen in Figure 7(b).

On the other hand, despite the fabrication process, which is very important in development of nanocomposites containing aligned CNTs in the direction of electric field, destructive factors such as bubble based voids can also significantly affect the overall properties of nanocomposites includes mechanical and electrical properties [38-43]. Formation of bubble-based voids in the thermoset resins, such as epoxy resin, can highly affect their structural properties

(a)
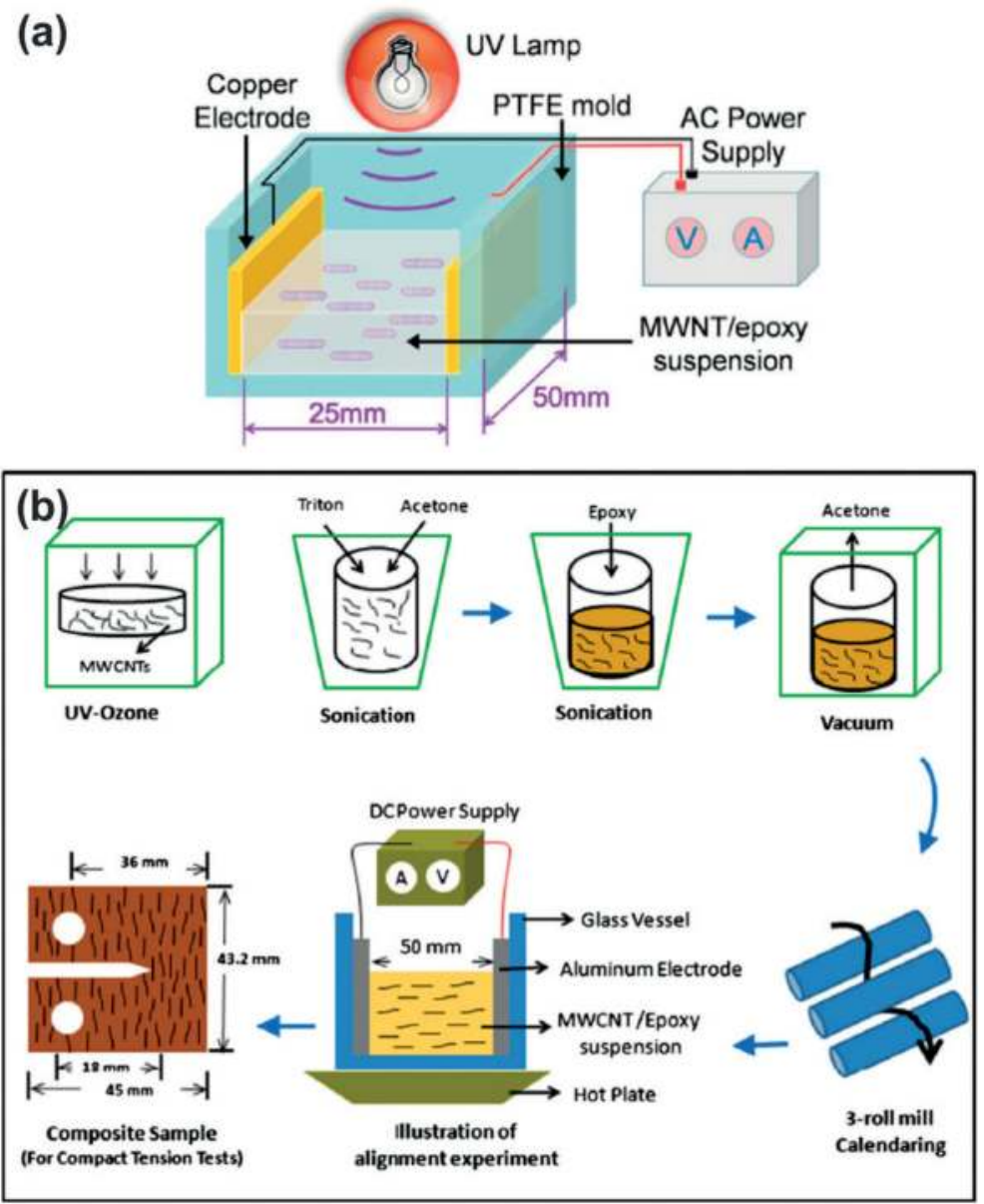

Figure 7. Production procedures presented by (a) Zhu et al. [7] and (b) Khan et al. [3]. 
and lead creation of stress concentration areas. Bubbles are created due to various factors; diffusion of air into the polymer matrix and amalgamation of small bubbles have the main role in the creation of large bubbles. After completion of matrix-curing steps, the bubbles transform to voids and due to higher internal pressure than pressure in border with matrix and matrix itself, thereby resulting in growth of cracks in radial direction around the void $[39,42,44]$. For this regard, researchers have developed various kind of techniques to minimize the overall amount of bubble based voids, including molecular dispersion of bubbles in the polymer matrix [39], simultaneous usage of vacuum and vibration [45], and vacuum shock technique [42].

Moreover, addition of curing agent to the epoxy resin can lead to generation of a great amount of bubbles throughout the matrix, which is due to the reaction between the resin and the curing agent and can be removed via vacuum shock technique before completion of the first curing step. For instance, the presence of bubble-based voids throughout the epoxy resin with $1.0 \mathrm{wt} \%$ randomly oriented single-walled carbon nanotube (SWCNTS) can lead to 63\% decrease in EMI shielding (X-band) and significant reduction in the electrical conductivity compared with nanocomposites holding small amount of bubble-based voids (less than $2 \%$ ) [42]. What is more, creation of moisture throughout the dielectric nanocomposites can lead to significant decrease in both dielectric constant and dielectric loss [46]. Absorbed moisture not only can lead to decrease in the electrical performance but also can deteriorate the reliability of the dielectric materials [47]. Thus, removal of moisture and bubbles from nanocomposites has high level of importance.

\section{Outcomes}

Alignment of CNTs within the matrix can lead to obvious anisotropy in different directions. In this regard, aligned network presented significant enhancement in mechanical and electrical properties for parallel direction compared to perpendicular direction and randomly distribution of CNTs within the matrix [2-4, 6-8, 13, 20, 35]. Khan et al. [3] achieved remarkably low percolation threshold and significant improvement in mechanical properties of nanocomposites containing aligned MWCNTs. In this case, low percolation threshold of $0.0031 \mathrm{vol} \%$ was obtained for parallel direction of CNT alignment, which is one order higher than $0.034 \mathrm{vol} \%$ corresponding to the randomly oriented CNTs or that measured in transverse direction of CNT alignment. It is worth noting that the conductivity of developed nanocomposites increased by about four orders of magnitude due to increase in CNT weight percentage from 0.001 to $0.01 \mathrm{wt} \%$, which shows a percolation behavior at even very low weight percentage of CNTs. Figure 8(a) shows the effect of CNT alignment on the electrical conductivity of developed nanocomposites containing aligned MWCNTs. As can be seen, alignment of CNTs within the matrix can lead to significant enhancement in the electrical conductivity, which is due to the increase in the interface of CNT bundles that enhanced the contact resistance $[27,48]$. This trend for parallel direction is significantly higher than perpendicular direction and randomly distributed CNTs. On application of electric field between electrodes, CNTs immediately align in the direction of electric field to form interconnected bundles even at a very low CNT content, which can lead to achievement of percolation in the direction of CNT alignment, while 
the similar trend is not occurred for perpendicular direction of CNT alignment at the same content [3]. Despite CNT content, there are some other effective factors which are also responsible for the anisotropy in percolation include applied voltage, polymerization time (cure time), applied temperature, and viscosity of the matrix [8]. Moreover, it should be mentioned that transverse migration and connection starts once the alignment in the longitudinal direction (parallel direction) completed. In this case, increase in CNT weight percentage can enhance the rate of transverse direction and thus minimize the differences between parallel and perpendicular directions. On the other hand, the same as electrical conductivity, alignment of CNTs within the matrix shows significant improvement in the mechanical properties of nanocomposites include tensile strength, storage modulus, and quasi-static fracture toughness, which indicating the string reinforcing effect of high-modulus CNTs. In this regard, alignment of MWCNTs within the matrix led to $40 \%$ and $15 \%$ improvement in the Young's modulus for nanocomposites containing $0.3 \mathrm{wt} \%$ aligned and unaligned MWCNT, respectively. However, it was observed that by further increase in the CNT concentration beyond $0.3 \mathrm{wt} \%$, overall mechanical properties of developed nanocomposites containing aligned MWCNTs decreased, which is due to the reduction in the degree of alignment at high CNT concentration. In fact, increase in the CNT concentration can lead to significant increase in the viscosity of matrix as well as fillers packing, which can lead to higher resistance and less available free spaces for CNTs in order to move and align in the direction of electric field. In Figure 8(b-d), effect of CNT alignment on storage modulus, Young's modulus, and quasi-static fracture toughness of nanocomposites can be seen [3].

On the other hand, present gaps and pores inside the aligned network due to low-weight percentage of CNTs can increase the contact resistance and thus reduce the electrical conductivity $[49,50]$. Alignment of CNTs can lead to significant drop in obtained resistivity for a percolation threshold [51]. Moreover, alignment of CNTs can avoid the entanglement among CNTs in a certain degree, which can avoid creation of the conductive path [31]. It was also reported that alignment of CNTs within the matrix can lead increase in the volume resistivity and thus decrease in the conductivity of matrix [52]. In a work by Gupta et al. [2], they have aligned CNTs with current-assisted technique, which was led to the formation of shortest, continuous path for the flow of electrons, and thus formation of a highly anisotropic conductive path. Obtained results showed that current passage assisted alignment of CNTs can lead to significant $360 \%$ improvement in the conductivity for parallel direction than random distribution. However, lower conductivity in transverse direction than randomly distributed structure has been seen, which shows the efficiency of alignment. Despite of that, CNTs are forming a liner path with minimal distance between the electrodes in order to assisting current directly pass through. Electric field-induced alignment recorded $28 \%$ increase in the conductivity for parallel direction than randomly distribution, while the transverse direction showed $58 \%$ decrease in the electrical conductivity. In fact, current-induced alignment resulted in creation of continuous channels of aligned CNT, where CNTs are contacted end to end with each other and formed and uninterrupted conductive pathway. In this case, electrons will get direct path of flow, which can lead to fantastic enhancement in the electrical conductivity.

Zhu et al. [7] reported that alignment of MWCNTs via AC electric field can lead to significant improvement in the storage modulus and electrical conductivity of developed nanocomposites. Their obtained results revealed that increase in concentration of MWCNTs as well as their alignment can lead significant enhancement in the storage modulus of developed nanocomposites. 

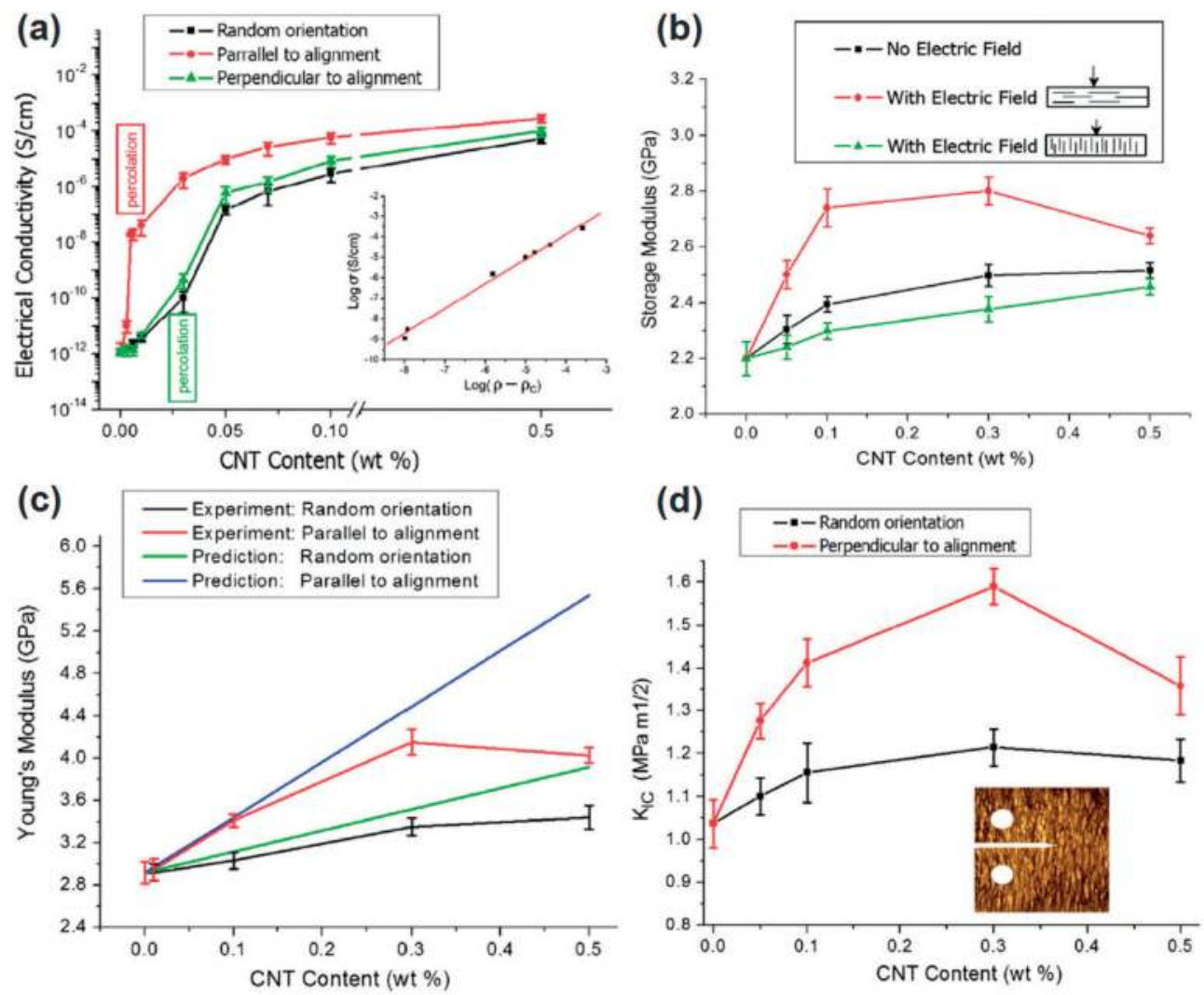

Figure 8. Effect of MWCNT alignment within the epoxy resin on (a) electrical conductivity, (b) storage modulus, (c) Young's modulus, and (d) quasi-static fracture toughness of developed nanocomposites [3].

(a)

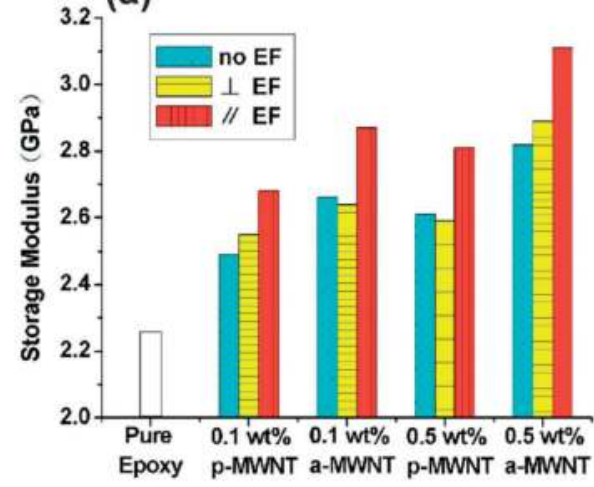

(b)

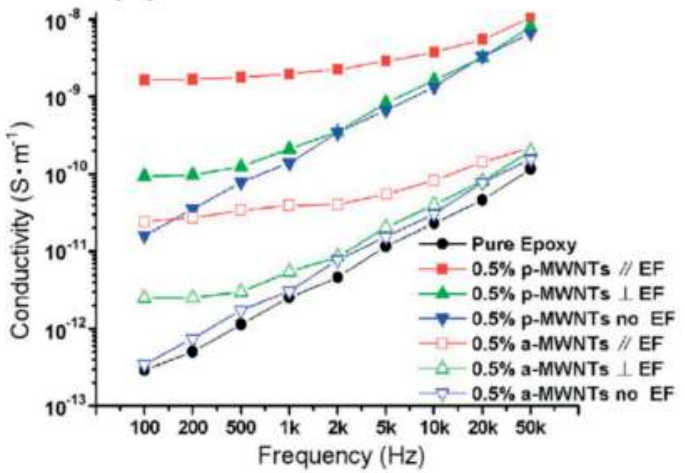

Figure 9. Effect of MWCNT alignment on (a) storage modulus and (b) electrical conductivity of developed nanocomposites [7]. 
Furthermore, nanocomposites containing amine functionalized MWCNTs (a-MWCNT) have shown higher storage modulus compared to pristine MWCNTs (p-MWCNT), which is due to the better compatibility and interaction between the matrix and a-MWCNTs. Moreover, measured storage modulus of aligned CNTs in parallel direction was greater than perpendicular direction and their randomly distribution within the matrix. On the other hand, nanocomposites containing p-MWCNT were presented higher electrical conductivity compared to a-MWCNT by about two orders of magnitude, which could be due to the decrease in length of a-MWCNT during chemical functionalization and increase in the a-MWCNT resistance. Figure 9(a) and (b) illustrated the effect of MWCNT alignment within the epoxy resin on storage modulus and electrical conductivity, respectively.

\section{Conclusions}

CNTs presenting extraordinary mechanical, thermal, and electrical properties, which have reinforcement of nanocomposites with various kinds of $\mathrm{CNT}$, can lead to significant improvement in their overall properties. In this case, application of electric field to the suspension containing CNT at different filler loadings can lead their alignment within the matrix and obvious anisotropy in different directions. In fact, CNTs act as path for transferring current from negative to positive electrode. This phenomenon can boost both electrical and mechanical properties of developed nanocomposites at same filler content. On the other hand, achieved results revealed that the overall properties of nanocomposites include mechanical and electrical properties are higher for parallel direction than perpendicular direction and random distribution of CNTs within the matrix, which is due to the desire of CNTs in formation of longitudinal connections than transverse connections.

Eventually, by alignment of CNTs within the matrix, significant improvement in overall properties of nanocomposites at same filler loadings compared with randomly distribution can be achieved, which is very essential for aerospace and aviation industries that encounter with serious limits in matter of structures' weight.

\section{Author details}

Ali Mohammad Amani ${ }^{1,2}$, Seyyed Alireza Hashemi ${ }^{1,2 *}$, Seyyed Mojtaba Mousavi ${ }^{1,2}$, Seyyed Milad Abrishamifar ${ }^{3}$ and Arash Vojood ${ }^{4}$

*Address all correspondence to: s.a.hashemi0@gmail.com

1 Department of Medical Nanotechnology, School of Advanced Medical Sciences and Technologies, Shiraz University of Medical Sciences, Shiraz, Iran

2 Pharmaceutical Sciences Research Center, Shiraz University of Medical Sciences, Shiraz, Iran

3 Department of Chemical Engineering, New York International University of Technology and Management, New York, USA

4 Young Researchers and Elite Club, Ardabil Branch, Islamic Azad University, Ardabil, Iran 


\section{References}

[1] Iijima S. Helical microtubules of graphitic carbon. Nature. 1991;354(6348):56

[2] Gupta P, Rajput M, Singla N, Kumar V, Lahiri D. Electric field and current assisted alignment of CNT inside polymer matrix and its effects on electrical and mechanical properties. Polymer. 2016;89:119-127

[3] Khan SU, Pothnis JR, Kim JK. Effects of carbon nanotube alignment on electrical and mechanical properties of epoxy nanocomposites. Composites Part A: Applied Science and Manufacturing. 2013;49:26-34

[4] Ma C, Zhang W, Zhu Y, Ji L, Zhang R, Koratkar N, Liang J. Alignment and dispersion of functionalized carbon nanotubes in polymer composites induced by an electric field. Carbon. 2008;46(4):706-710

[5] Mecklenburg M, Mizushima D, Ohtake N, Bauhofer W, Fiedler B, Schulte K. On the manufacturing and electrical and mechanical properties of ultra-high wt.\% fraction aligned MWCNT and randomly oriented CNT epoxy composites. Carbon. 2015;91:275-290

[6] Wang Q, Dai J, Li W, Wei Z, Jiang J. The effects of CNT alignment on electrical conductivity and mechanical properties of SWNT/epoxy nanocomposites. Composites Science and Technology. 2008;68(7):1644-1648

[7] Zhu YF, Ma C, Zhang W, Zhang RP, Koratkar N, Liang J. Alignment of multiwalled carbon nanotubes in bulk epoxy composites via electric field. Journal of Applied Physics. 2009;105(5):054319

[8] Zhang RP, Zhu YF, Ma C, Liang J. Alignment of carbon nanotubes in poly (methyl methacrylate) composites induced by electric field. Journal of Nanoscience and Nanotechnology. 2009:9(5):2887-2893

[9] Prolongo S, Meliton B, Del Rosario G, Ureña A. New alignment procedure of magnetiteCNT hybrid nanofillers on epoxy bulk resin with permanent magnets. Composites Part B: Engineering. 2013;46:166-172

[10] Hobbie EK, Wang H, Kim H, Han CC, Grulke E, Obrzut J. Optical measurements of structure and orientation in sheared carbon-nanotube suspensions. Review of Scientific Instruments. 2003;74(3):1244-1250

[11] Vigolo B, Penicaud A, Coulon C, Sauder C, Pailler R, Journet C, Bernier P, Poulin P. Macroscopic fibers and ribbons of oriented carbon nanotubes. Science. 2000;290(5495): 1331-1334

[12] Haggenmueller R, Gommans H, Rinzler A, Fischer JE, Winey K. Aligned single-wall carbon nanotubes in composites by melt processing methods. Chemical Physics Letters. 2000;330(3):219-225

[13] Thostenson ET, Chou TW. Aligned multi-walled carbon nanotube-reinforced composites: Processing and mechanical characterization. Journal of Physics D: Applied Physics. 2002;35(16):L77 
[14] Ko F, Gogotsi Y, Ali A, Naguib N, Ye H, Yang G, Li C, Willis P. Electrospinning of continuous carbon nanotube-filled nanofiber yarns. Advanced Materials. 2003;15(14): 1161-1165

[15] Sen R, Zhao B, Perea D, Itkis ME, Hu H, Love J, Bekyarova E, Haddon RC. Preparation of single-walled carbon nanotube reinforced polystyrene and polyurethane nanofibers and membranes by electrospinning. Nano Letters. 2004;4(3):459-464

[16] Monti M, Natali M, Torre L, Kenny JM. The alignment of single walled carbon nanotubes in an epoxy resin by applying a DC electric field. Carbon. 2012;50(7):2453-2464

[17] Martin C, Sandler J, Windle A, Schwarz MK, Bauhofer W, Schulte K, Shaffer M. Electric field-induced aligned multi-wall carbon nanotube networks in epoxy composites. Polymer. 2005;46(3):877-886

[18] Hsu H, Sharma N, Ruoff R, Patankar N. Electro-orientation in particle light valves. Nanotechnology. 2005;16(2):312

[19] Abbasi S, Carreau PJ, Derdouri A. Flow induced orientation of multiwalled carbon nanotubes in polycarbonate nanocomposites: Rheology, conductivity and mechanical properties. Polymer. 2010;51(4):922-935

[20] Arjmand M, Mahmoodi M, Gelves GA, Park S, Sundararaj U. Electrical and electromagnetic interference shielding properties of flow-induced oriented carbon nanotubes in polycarbonate. Carbon. 2011;49(11):3430-3440

[21] Lehman JH, Terrones M, Mansfield E, Hurst KE, Meunier V. Evaluating the characteristics of multiwall carbon nanotubes. Carbon. 2011;49(8):2581-2602

[22] Yoo HJ, Lee SY, You NH, Lee DS, Yeo H, Choi YM, Goh M, Park J, Akagi K, Cho JW. Dispersion and magnetic field-induced alignment of functionalized carbon nanotubes in liquid crystals. Synthetic Metals. 2013;181:10-17

[23] Sarno M, Sannino D, Leone C, Ciambelli P. CNTs tuning and vertical alignment in anodic aluminium oxide membrane. Journal of Natural Gas Chemistry. 2012;21(6):639-646

[24] Han Y, Li S, Chen F, Zhao T. Multi-scale alignment construction for strong and conductive carbon nanotube/carbon composites. Materials Today Communications. 2016;6:56-68

[25] Arjmand M, Apperley T, Okoniewski M, Sundararaj U. Comparative study of electromagnetic interference shielding properties of injection molded versus compression molded multi-walled carbon nanotube/polystyrene composites. Carbon. 2012;50(14):5126-5134

[26] Mahmoodi M, Arjmand M, Sundararaj U, Park S. The electrical conductivity and electromagnetic interference shielding of injection molded multi-walled carbon nanotube/ polystyrene composites. Carbon. 2012;50(4):1455-1464

[27] Han B, Xue X, Xu Y, Zhao Z, Guo E, Liu C, Luo L, Hou H. Preparation of carbon nanotube film with high alignment and elevated density. Carbon. 2017;122:496-503

[28] Chapkin WA, McNerny DQ, Aldridge MF, He Y, Wang W, Kieffer J, Taub AI. Real-time assessment of carbon nanotube alignment in a polymer matrix under an applied electric field via polarized Raman spectroscopy. Polymer Testing. 2016;56:29-35 
[29] Donovan K, Scott K. Transient electric birefringence in suspensions of single-walled carbon nanotubes. Physical Review B. 2005;72(19):195432

[30] Robb-Smith T, Donovan K, Scott K, Somerton M. Induced electro-optic effects in singlewalled carbon nanotubes. I. Polarizability of metallic nanotubes. Physical Review B. 2011;83(15):155414

[31] Cheng J, Pu H. Influences of matrix viscosity on alignment of multi-walled carbon nanotubes in one-dimensional confined space. European Polymer Journal. 2017;89:431-439

[32] Blatt S, Hennrich F, Löhneysen HV, Kappes MM, Vijayaraghavan A, Krupke R. Influence of structural and dielectric anisotropy on the dielectrophoresis of single-walled carbon nanotubes. Nano Letters. 2007;7(7):1960-1966

[33] Oliva-Avilés A, Avilés F, Sosa V, Seidel G. Dielectrophoretic modeling of the dynamic carbon nanotube network formation in viscous media under alternating current electric fields. Carbon. 2014;69:342-354

[34] Ma PC, Siddiqui NA, Marom G, Kim JK. Dispersion and functionalization of carbon nanotubes for polymer-based nanocomposites: A review. Composites Part A: Applied Science and Manufacturing. 2010;41(10):1345-1367

[35] Xie XL, Mai YW, Zhou XP. Dispersion and alignment of carbon nanotubes in polymer matrix: A review. Materials Science and Engineering: R: Reports. 2005;49(4):89-112

[36] Sham ML, Kim JK. Surface functionalities of multi-wall carbon nanotubes after UV/ Ozone and TETA treatments. Carbon. 2006;44(4):768-777

[37] Kamat PV, Thomas KG, Barazzouk S, Girishkumar G, Vinodgopal K, Meisel D. Selfassembled linear bundles of single wall carbon nanotubes and their alignment and deposition as a film in a dc field. Journal of the American Chemical Society. 2004;126(34): 10757-10762

[38] Liu L, Zhang BM, Wang DF, Wu ZJ. Effects of cure cycles on void content and mechanical properties of composite laminates. Composite Structures. 2006;73(3):303-309

[39] Li Y, Li Q, Ma H. The voids formation mechanisms and their effects on the mechanical properties of flax fiber reinforced epoxy composites. Composites Part A: Applied Science and Manufacturing. 2015;72:40-48

[40] Jongwoo P, Taweeplengsangsuke J, Theis C, Osenbach J. Epoxy adhesive used in optical fiber/passive component: Kinetics voids and reliability. Proceedings 51st Electronic Components and Technology Conference; 2001. pp. 637-644

[41] Hernández S, Sket F, Molina-Aldareguı J, González C, Lorca J. Effect of curing cycle on void distribution and interlaminar shear strength in polymer-matrix composites. Composites Science and Technology. 2011;71(10):1331-1341

[42] Hashemi SA, Mousavi SM. Effect of bubble based degradation on the physical properties of single wall carbon nanotube/epoxy resin composite and new approach in bubbles reduction. Composites Part A: Applied Science and Manufacturing. 2016;90:457-469 
[43] Bodaghi M, Cristóvão C, Gomes R, Correia N. Experimental characterization of voids in high fibre volume fraction composites processed by high injection pressure RTM. Composites Part A: Applied Science and Manufacturing. 2016;82:88-99

[44] Nie L, Lee K, Lee S, Shi T, Liao G. Void control in adhesive bonding using thermosetting polymer. Sensors and Actuators A: Physical. 2011;167(2):398-405

[45] Muric-Nesic J, Compston P, Noble N, Stachurski Z. Effect of low frequency vibrations on void content in composite materials. Composites Part A: Applied Science and Manufacturing. 2009;40(4):548-551

[46] Chao F, Bowler N, Tan X, Liang G, Kessler MR. Influence of adsorbed moisture on the properties of cyanate ester $/ \mathrm{BaTiO}_{3}$ composites. Composites Part A: Applied Science and Manufacturing. 2009;40(8):1266-1271

[47] Cheng YL, Leon KW, Huang JF, Chang WY, Chang YM, Leu J. Effect of moisture on electrical properties and reliability of low dielectric constant materials. Microelectronic Engineering. 2014;114:12-16

[48] Zhao S, Zheng Z, Huang Z, Dong S, Luo P, Zhang Z, Wang Y. Cu matrix composites reinforced with aligned carbon nanotubes: Mechanical, electrical and thermal properties. Materials \& Design. 2016;675:82-91

[49] Jakubinek MB, Johnson MB, White MA, Jayasinghe C, Li G, Cho W, Schulz MJ, Shanov $\mathrm{V}$. Thermal and electrical conductivity of array-spun multi-walled carbon nanotube yarns. Carbon. 2012;50(1):244-248

[50] Miao M. Electrical conductivity of pure carbon nanotube yarns. Carbon. 2011;49(12): 3755-3761

[51] Cheng J, Pu H, Du J. A processing method with high efficiency for low density polyethylene nanofibers reinforced by aligned carbon nanotubes via nanolayer coextrusion. Polymer. 2017;111:222-228

[52] Li Y, Zhao L, Shimizu H. Electrically conductive polymeric materials with high stretchability and excellent elasticity by a surface coating method. Macromolecular Rapid Communications. 2011;32(3):289-294 